\title{
Author Index Vol. 47, 1995
}

Ackermann, H. 15 Alm,N. 165 Bierens, E. 252 Bougie, F. 24 Boyanov, B. 291 Brassard, C. 39 Bremerich, A. 193 Buekers, R. 252 Culatta,R. 1 Dalkvist,J. 262 Dejonckere, P.H. 199 Dekker,J. 199 Doskov,D. 291 Eggeling, V. 193 Eufinger,H. 193 Gellrich, N.-C. 193 Gerull,G. 210 Heaton, J.M. 286 Hehr,T. 15 Hertrich, I. 15 Hofmann, G. 236 Ivanov, T. 291 Johannsen, H.S. 33 Kaiser, E. 242 Kingma, H. 252 Lacy, P. 286 Lass,NJ. 247 Lastovka,M. 318 Laukkanen, A.-M. 324,331 Le Dorze, G. 24, 39 Leeper, L.H. 1 Lever, N. 39 Lindholm,P. 324,331 Logemann, J.A. 140

Luotonen, M. 310 McAllister, A. 262 Marres, E.H.M.A. 252 Middleton, G.F. 247 Mrowinski, D. 210 Novak, A. 279 Pabst,F. 236 Pahn,E. 229 Pahn,J. 229 Pannbacker, M. 247 Parker, A.J. 286 Parker, G. 286 Parnes,P. 165 Perelló,J. 243 Raaijmakers, M.F. 199 Ruscello, D.M. 247 Rustin,L. 123 Ryalls, J. 24, 39 Schaerlaekens, A. 303 Schmitt,J.F. 247 Schneider, C. 210 Schweppenheiser, K. 247 Sederholm, E. 262 Sieron, J. 33 Sundberg,J. 218,262 Tymnik,G 236 Vilkman,E. 324,331 Vokrál, J. 279 Westphal, K.P. 33 Wilke,W. 229 Yardley,M. 286 Zee, J. van der 199 Zieger,K. 210 